\title{
Développement d'une liaison entre une briquette en composite carbone/carbone et une structure de refroidisseur en cuivre
}

\author{
Jacques Schlosser $^{a}$, Jean-Jacques Cordier, Raphä̈l Mitteau et Alain Durocher \\ Association EURATOM-CEA, DSM/DRFC/SIPP, CEA Cadarache 13108 St Paul lez Durance Cedex, France
}

Reçu le 2 juillet 2004, accepté le 5 novembre 2004

\begin{abstract}
Résumé - La liaison entre une tuile en composite carbone/carbone (CFC) et un substrat métallique est du même type qu'une liaison céramique/métal. Elle est difficile à réaliser du fait des différences de dilatation entre les matériaux ce qui induit des contraintes internes très importantes. Pourtant dans la machine de fusion contrôlée Tore Supra (CEA Cadarache, 13108 St Paul Lez Durance, France) ce type de liaison, développée avec succès depuis de nombreuses années, a permis d'aboutir à la fabrication d'un limiteur torö̈dal de $7,6 \mathrm{~m}^{2}$, ce qui représente la réalisation de jonctions $\mathrm{CFC} / \mathrm{métal}$ de plus de 12000 tuiles. Le procédé utilisé consiste en une structuration géométrique de la surface de CFC à joindre, par un traitement laser, ce qui permet un bon accrochage mécanique de la tuile avec la brasure ou le métal fondu.
\end{abstract}

Mots clés : Fusion contrôlée / composants face au plasma / dilatations différentielles / assemblages carbone-cuivre / active metal casting

\begin{abstract}
Bonding developpement between a tile made of carbon fibre composite and a copper cooling structure. The bond between a tile made of carbon fibre composite (CFC) and a metallic heat sink is similar to a ceramic/metal bond. It is difficult to perform due to the mismatch between the material expansions, which create high internal stresses. However in the Tore Supra controlled fusion machine (CEA Cadarache, 13108 St Paul Lez Durance, France), this kind of bond, in development since many years, has allowed a $7.6 \mathrm{~m}^{2}$ toroidal limiter to be achieved, which led to perform $\mathrm{CFC} / \mathrm{metal}$ bonds for more than 12000 tiles. The used process consists of a geometrical structuring of the CFC surface to be joined, using a laser treatment, which allows a good mechanical coupling of the tile to the brazing material or cast metal.
\end{abstract}

Key words: Controlled fusion / plasma facing components / mismatch of thermal expansions / carbon-copper bonding / active metal casting

\section{Introduction}

La machine de Fusion Contrôlée Tore Supra est le seul Tokamak qui a utilisé, depuis son démarrage en 1988, des composants face au plasma (CFP) activement refroidis à l'eau. Certains de ces éléments internes sont aujourd'hui dimensionnés pour des flux convectifs pouvant atteindre jusqu'à $15 \mathrm{MW} \cdot \mathrm{m}^{-2}$, la machine étant conçue pour des plasmas de plusieurs minutes.

Ces éléments sont recouverts de tuiles de carbone, matériau qui supporte bien les hautes températures, qui a une bonne conductivité thermique et qui ne pollue pas le plasma.

Plusieurs générations de ces composants face au plasma se sont succédées dans la machine. La fabrication

\footnotetext{
${ }^{a}$ Auteur correspondant : jacques.schlosser@cea.fr
}

de ces éléments est un défi technologique du fait des contraintes résiduelles, dues aux dilatations différentielles qui se développent lors de l'assemblage des briquettes sur leur support métallique. Ces problèmes ont nécessité de nombreux développements qui ont permis d'arriver à la génération actuelle développée dans les années 90.

La dernière fabrication a néanmoins conduit à un taux de fissuration de tuiles faible (3 à $5 \%$ ) mais inacceptable conduisant à de nombreux rejets d'éléments et à des retards importants dans la fabrication.

Après 4 ans de fabrication un limiteur complet de plus de 12000 tuiles a pu être installé avec succès dans la machine début 2002 et a déjà permis de réaliser des plasmas de longue durée constituant des records mondiaux encore jamais atteints. 


\section{Nomenclature}

\begin{tabular}{|ll|}
\hline$\alpha$ & coefficient de dilatation $\left(\mathrm{K}^{-1}\right)$ \\
$\sigma_{y y}, \sigma_{x x}, \sigma_{z z}, \sigma_{x y}, \sigma_{x z}, \sigma_{y z}$ & conductivité thermique $\left(\mathrm{W} \cdot \mathrm{m}^{-1} \cdot \mathrm{K}^{-1}\right)$ \\
$\theta$ & valeur de l'angle polaire (radian) \\
$r$ & distance au point d'intersection interface/bord-libre (m) \\
$w$ & ordre de la singularité \\
$E$ & Module d'Young (GPa) \\
$K_{\mathrm{I}}$ & coefficient d'intensification en mode I (traction) $\left(\mathrm{Mpa} \cdot \mathrm{m}^{1 / w}\right)$ \\
$K_{\mathrm{II}}$ & coefficient d'intensification en mode II (cisaillement) $\left(\mathrm{Mpa}^{1 / w}\right)$ \\
$R_{\mathrm{m}}$ & valeurs de la résistance à la traction pour un matériau isotrope $(\mathrm{MPa})$ \\
$R_{\mathrm{cis}}$ & valeurs de la résistance au cisaillement pour un matériau isotrope $(\mathrm{MPa})$ \\
$R_{x}, R_{y}, R_{z}$ & valeurs des résistances à la traction (MPa) \\
$R_{x^{\prime}}, R_{y^{\prime}}, R_{z^{\prime}}$ & valeurs des résistances à la compression $(\mathrm{MPa})$ \\
$S_{x y}, S_{x z}, S_{y z}$ & valeurs des résistances au cisaillement (MPa) \\
\hline
\end{tabular}

Nous abordons successivement dans ce papier la présentation de la machine Tore Supra et de la problématique des composants face au plasma, la description des différentes générations de composants développés et notamment les problèmes rencontrés lors du développement et de la fabrication de la dernière génération.

\section{La machine de fusion Tore Supra}

Tore Supra est une machine appelée Tokamak, la chambre à vide a une forme torique avec un grand rayon $R=2,3 \mathrm{~m}$ et un petit rayon $a=0,8 \mathrm{~m}$ (Fig. 1). Elle permet de réaliser des plasmas de fusion au deutérium par confinement magnétique : un solénö̈de central et 6 grandes bobines horizontales, extérieures à la chambre à vide, forment l'aimant poloïdal à bobinage en cuivre, 18 bobines verticales intégrées dans la double paroi de la chambre à vide forment l'aimant toroïdal, ce dernier utilise des brins supra conducteurs de $\mathrm{NbTi}$ et fonctionne à une température de $1,7 \mathrm{~K}$. Le champ toroïdal est de 4,5 $\mathrm{T}$ au centre du tore. Le courant plasma (1 à 1,5 MA) est d'abord induit par variation de courant dans les bobines puis est ensuite maintenu grâce à de la puissance injectée sous forme de micro ondes. Contrairement aux autres machines de fusion qui expérimentent des plasmas de courte durée (quelques secondes) et fonctionnent sur le mode inertiel (la chaleur accumulée sur les parois est évacuée entre les plasmas sur des temps longs), la machine Tore Supra a été conçue pour fonctionner avec des plasmas de longue durée (plusieurs minutes) sur un mode continu. Cela conduit à avoir, outre des bobines supraconductrices, des parois activement refroidies qui fonctionnent à l'état d'équilibre. Les éléments de Tore Supra sont alimentés par une boucle d'eau à $3,5 \mathrm{MPa}$ et $150{ }^{\circ} \mathrm{C}$. La machine permet d'explorer la physique des plasmas dans des domaines où certains paramètres ou constantes de temps ne peuvent plus être négligés. Un plasma d'une minute a été obtenu en 1992, puis un plasma de 2 min a été réalisé en 1996 avec une énergie de plasma de 280 MJ, ce qui constituait un record absolu. En début d'année 2002 un nouveau record a été atteint avec un plasma de $3 \mathrm{~min} 30 \mathrm{~s}$ et une énergie de $600 \mathrm{MJ}$, encore amélioré en août 2002, avec un plasma de $4 \mathrm{~min} 23 \mathrm{~s}$ et $750 \mathrm{MJ}$ (Fig. 2). Ces résultats n'ont pu être obtenus que grâce à la mise en place de la $3^{\mathrm{e}}$ génération de CFP.

\section{La problématique des composants face au plasma}

Certains des composants face au plasma vont recevoir des flux convectifs de particules (ions) très intenses conduisant à des flux de chaleur de l'ordre de 1 à $15 \mathrm{MW} \cdot \mathrm{m}^{-2}\left(0.1\right.$ à $\left.1,5 \mathrm{~kW} \cdot \mathrm{cm}^{-2}\right)$. Sous l'impact des ions des particules de matière vont être arrachées tandis que les surfaces des composants vont atteindre des températures très élevées. Afin de ne pas polluer le plasma on a le choix entre des matériaux relativement tendres et à faible numéro atomique : bore, béryllium, carbone ou bien des matériaux très durs et lourds type tungstène. Dans la machine Tore Supra, on a opté pour des briquettes de carbone. C'est un matériau qui supporte bien les hautes températures sous vide (sublimation sous vide à des températures supérieures à $2000{ }^{\circ} \mathrm{C}$ ) et il est relativement bon conducteur de la chaleur $\left(60 \mathrm{~W} \cdot \mathrm{m}^{-1} \cdot \mathrm{K}^{-1}\right.$ sous forme polycristallin et $150 \mathrm{~W} \cdot \mathrm{m}^{-1} \cdot \mathrm{K}^{-1}$ sous forme de composite à fibres de carbone).

Dès l'origine le graphite polycristallin est utilisé dans Tore Supra avec une technique d'assemblage par brasage. Deux types de brasures sont utilisés le TiCuSil ( Ti-Cu-Ag) et le $\mathrm{TiCuNi}$, la présence de titane étant indispensable pour un bon accrochage sur le carbone par l'intermédiaire des carbures de Ti formés. Ces brasures nécessitent d'opérer sous vide et à $950{ }^{\circ} \mathrm{C}$. Malheureusement les dilatations différentielles entre la briquette et le support sont très importantes $\left(5 \times 10^{-6} \mathrm{~K}^{-1}\right.$ pour le 


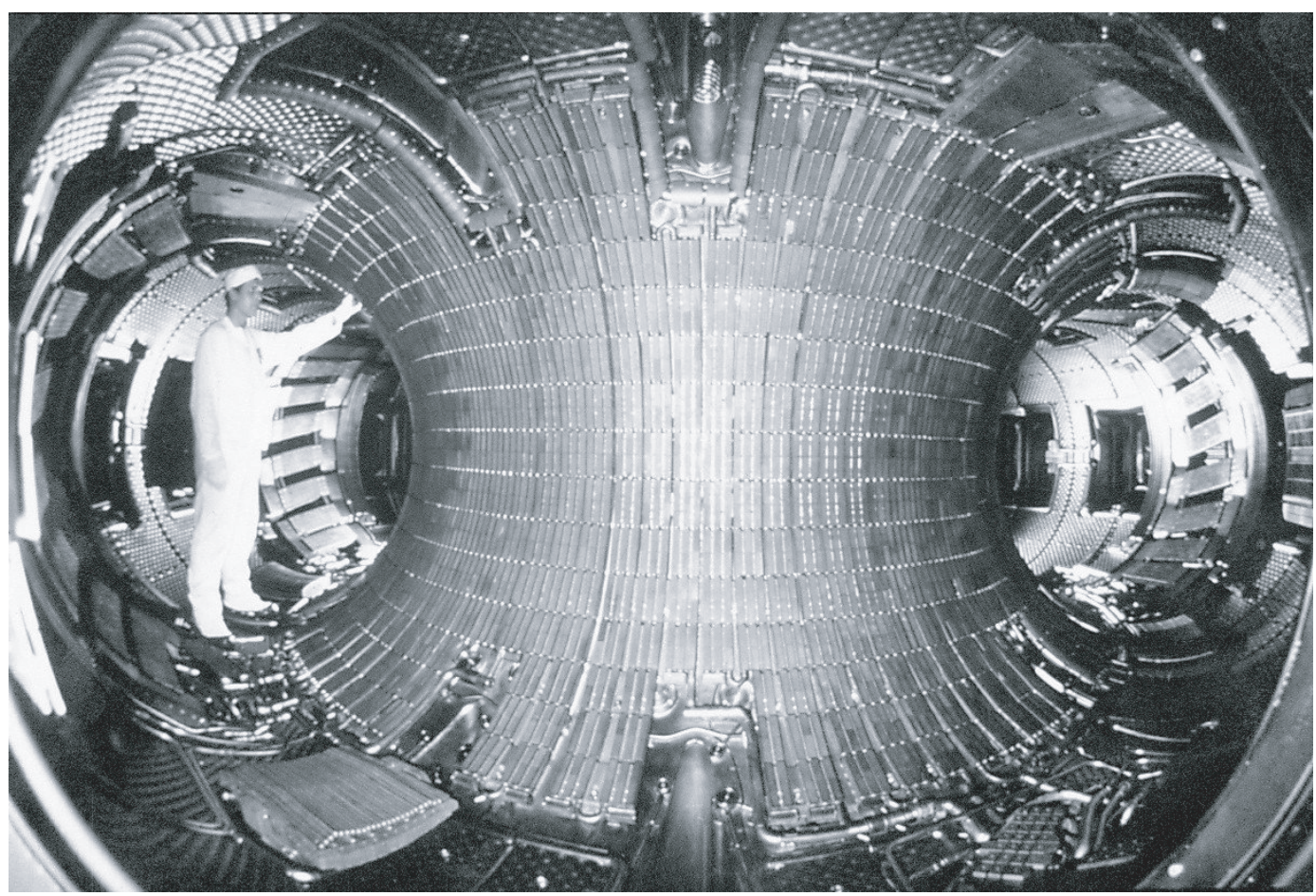

Fig. 1. Vue intérieure de la machine Tore Supra montrant les composants internes dans la configuration initiale. L'image montre 1) la 1ère paroi interne qui forme un limiteur, véritable ceinture de protection dans la partie à fort champ, 2) un des 6 limiteurs verticaux à gauche, 3) la protection externe en inox gaufré, elle aussi activement refroidie (visible en haut à $\mathrm{G}$ et derrière le technicien).

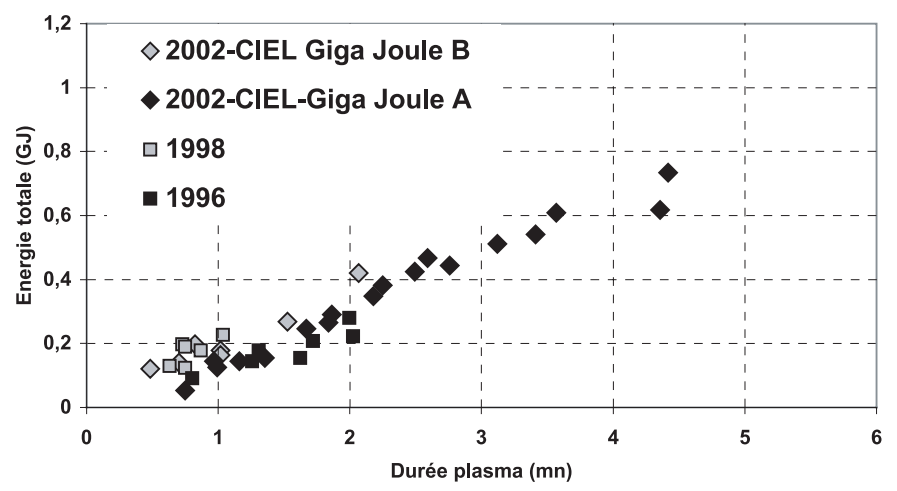

Fig. 2. Comparaison des plasmas obtenus en temps et en énergie entre les années 96-98 et l'année 2002. Le record atteint est aujourd'hui de 4 min 23 s et 750 MJ d'énergie injectée (ce qui correspond à une puissance moyenne de 2,85 MW).

carbone polycristallin et 18 à $20 \times 10^{-6} \mathrm{~K}^{-1}$ pour le support métallique) (Fig. 3). Ce problème est bien connu notamment pour les liaisons céramique-métal. À la fin du refroidissement de 900 à $20{ }^{\circ} \mathrm{C}$ de l'assemblage, le carbone est globalement en compression $\left(\sigma_{x x}\right)$, mais une singularité des contraintes existe à l'interface carbone-métal au niveau du bord libre [1]. Cette singularité peut s'écrire [2] :

$$
\left(\sigma_{y y}-i \sigma_{x y}\right)_{\theta=0}=\frac{K_{\mathrm{I}}-i K_{\mathrm{II}}}{\sqrt{2 \pi} r^{\omega}}+\sigma_{0}
$$

assemblage à haute température

déformée à température ambiante

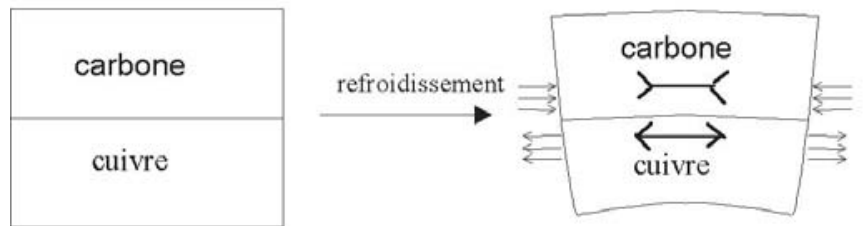

Fig. 3. Naissance de contraintes résiduelles dans un assemblage carbone/cuivre lors du refroidissement après brasage, le carbone se trouve en compression tandis que le cuivre est en traction, l'ensemble se déforme en forme de dôme.

L'ordre de la singularité $\omega$ dépend de l'angle du bord libre avec l'interface et du rapport des modules d'Young comme montré dans la littérature [3]. Dans le cas de la liaison carbone-cuivre le rapport du module Young du carbone sur celui du cuivre est de l'ordre de 0,1 à 0,17 .

\section{Les différentes générations de CFP dans Tore Supra}

Le premier carbone sélectionné pour Tore Supra en 1985 était un carbone à grains fins (polycristallin 5890PT de Carbone Lorraine) $\left(\lambda=73 \mathrm{~W} \cdot \mathrm{m}^{-1} \cdot \mathrm{K}^{-1}, \alpha=4,5 \times\right.$ $\left.10^{-6} \mathrm{~K}^{-1}, E=13 \mathrm{GPa}, R_{\mathrm{m}}=34 \mathrm{MPa}, R_{\text {cis }}=21 \mathrm{MPa}\right)$. Rapidement le brasage de ce type de matériau sur un tube support en inox ou en cuivre durci ( $\mathrm{CuCrZr})$ s'est 


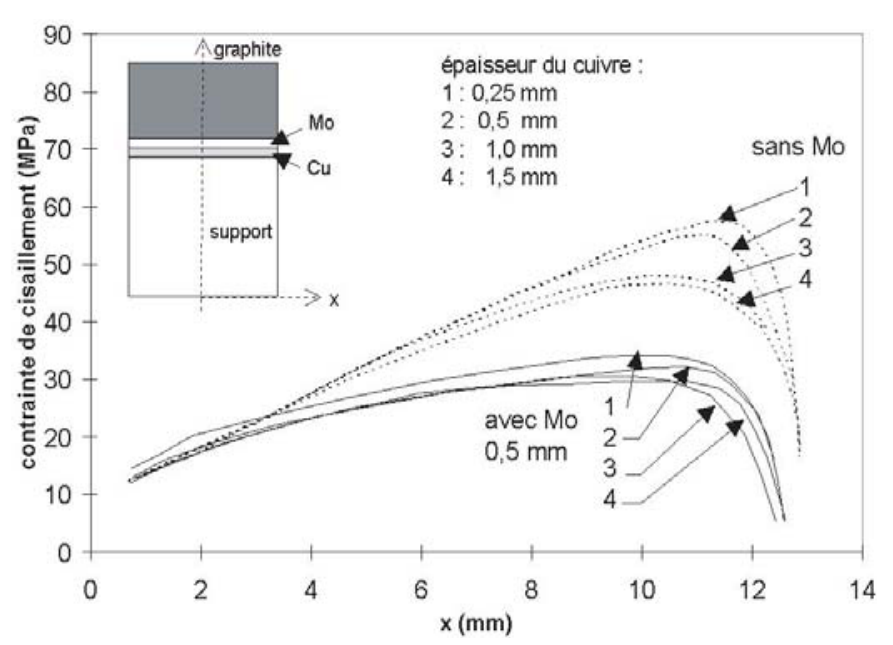

Fig. 4. Calcul des contraintes de cisaillement à la fin du cycle de brasage dans le graphite à $0,5 \mathrm{~mm}$ au-dessus de l'interface en fonction de l'épaisseur de la couche d'adaptation en cuivre (les contraintes sont extrapolées sur les points du maillage à partir des valeurs obtenues aux points d'intégration). Le calcul est effectué en déformation plane généralisée sur une moitié de la pièce avec les conditions aux limites nécessaires pour le blocage du support en déplacement $X$ et $Y$ (support $23 \times$ $23 \mathrm{~mm}$, couche de molybdène $0,5 \mathrm{~mm}$, tuile $10 \mathrm{~mm}$ ). Le calcul montre bien l'intérêt d'une couche de molybdène dans ce cas.

révélé très laborieux. Lors du brasage, du fait du rétreint du matériau métallique, la briquette se met en forme de dôme; elle est fortement sollicitée en flexion et en cisaillement au niveau de l'interface (Fig. 3). De façon à décharger par plastification les contraintes de dilatations différentielles une couche d'adaptation en cuivre doux de $1 \mathrm{~mm}$ d'épaisseur a été introduite. Cependant, à elle seule, cette technique ne suffisait pas à empêcher la fissuration des briquettes. Une couche de renforcement de $0,5 \mathrm{~mm}$ de molybdène a alors été introduite ce qui a permis de braser avec succès près de $95 \%$ des briquettes. L'avantage du molybdène, dans ce cas, est d'avoir un coefficient de dilatation thermique quasiment identique au carbone polycristallin $\left(\alpha=5,6 \times 10^{-6} \mathrm{~K}^{-1}\right)$. Un calcul par éléments finis avec un cuivre plastique montre bien, sans couche de molybdène, des contraintes de cisaillement inacceptables pour un matériau qui ne peut guère supporter plus de 20 à $25 \mathrm{MPa}$ alors que ces contraintes sont presque diminuées d'un facteur 2 avec la couche de molybdène (Fig. 4). De cette façon près de 7500 tuiles, de $10 \mathrm{~mm}$ d'épaisseur, ont été brasées pour la première paroi interne de Tore Supra en 1986. Cette première paroi couvre $12 \mathrm{~m}^{2}$ de l'enceinte (Fig. 1). De même, près de 5500 tuiles semi-circulaires ont été brasées en 1989 sur des tubes en alliages de cuivre pour la fabrication de 6 limiteurs verticaux (Fig. 5) [4].

Avec la disponibilité de nouveaux matériaux type composite carbone à fibres de carbone (CFC) une $2^{\mathrm{e}}$ génération de CFP a été développée dans les années 95-96. Le renforcement du carbone étant assuré par les fibres, la couche de molybdène a pu être supprimée et la couche de cuivre a été portée à $2 \mathrm{~mm}$. Le procédé de brasage, sur des tubes inox, confié à la société Plansee en Autriche (Reutte, Tirol), a été amélioré par cet industriel (Fig. 6), compte tenu de son expérience, acquise dans la fabrication des cathodes tournantes des tubes de rayons X (brasage graphite/tungstène). À part quelques rares défauts de brasage détectés à la fabrication aucune tuile ne s'est fissurée pendant la fabrication ou durant le fonctionnement dans la machine $[5,6]$.

À partir de 1995 une $3^{\mathrm{e}}$ génération de CFP a été développée en étroite collaboration avec la société Plansee. Le cycle de brasage à haute température étant peu compatible avec le support refroidi en alliage de cuivre durci ( $\mathrm{CuCrZr})$, un processus de liaison sans brasage a été mis au point : un traitement laser de la surface est d'abord effectué comme dans la génération précédente (réseau de micro trous avec un pas de 0,25 à $0,5 \mathrm{~mm}$ ), un dépôt de titane est ensuite effectué à très haute température de façon à former des carbures de titane, puis une couche de cuivre est coulée sur la surface et enfin la tuile avec sa semelle de cuivre est soudée par faisceau d'électrons sur le support en $\mathrm{CuCrZr}$ [7]. Le procédé a été breveté sous le nom d'AMC ${ }_{\AA}$ (Active Metal Casting). Ce procédé a permis de réaliser 200 éléments de limiteurs d'antennes (Fig. 7) [8] et 600 éléments pour le limiteur pompé toroïdal [9-11] (Figs. 8 et 9). Durant la phase de développement les éléments sont testés en fatigue dans une station à haut flux thermiques [12]. Lors de la réception des composants, la réponse thermique de chaque briquette est contrôlée sur un banc de test infrarouge [13].

\section{Caractérisation du CFC et du joint AMC}

Lors de la fabrication des éléments du limiteur pompé toroidal de nombreuses tuiles se sont fissurées, contrairement à la fabrication des éléments de protection d'antennes qui s'était déroulée sans problèmes majeurs. Ces défauts ont été attribués à la variabilité de la porosité du CFC qui peut avoir des conséquences sur le dépôt de titane ainsi qu'à l'utilisation d'un $2^{\mathrm{e}}$ lot de CFC lequel s'est révélé moins «flexible» (module d'Young plus élevé) et avec un allongement à la rupture moindre donc moins adaptable aux déformations différentielles [10].

On observe 2 types de fissures qui se développent à l'interface $\mathrm{CFC} /$ cuivre, l'une que l'on peut attribuer à la contrainte de traction et qui se caractérise par un délaminage CFC/Cuivre (Fig. 10) l'autre que l'on peut attribuer à la contrainte de cisaillement et qui se caractérise par un cisaillement des picots de cuivre (Fig. 11) [14,15].

La caractérisation du CFC et du joint $\mathrm{CFC} /$ cuivre a fait l'objet d'une étude spécifique en 97-99 [16]. Le CFC est un matériau composite à comportement sensiblement non-linéaire mais qui a tendance à « s'assouplir » au cours des chargements pour retrouver un caractère linéaire à module d'Young moins élevé. Un critère de rupture de type Tsai-Wu s'est montré satisfaisant :

$$
\begin{aligned}
F_{11} \sigma_{x x}^{2}+F_{22} \sigma_{y y}^{2}+F_{33} \sigma_{z z}^{2}+C_{12} \sigma_{x y}^{2}+C_{13} \sigma_{x z}^{2}+C_{23} \sigma_{y z}^{2} \\
+H_{11} \sigma_{x x}+H_{22} \sigma_{y y}+H_{33} \sigma_{z z} \\
+F_{12} \sigma_{x x} \sigma_{y y}+F_{13} \sigma_{x x} \sigma_{z z}+F_{23} \sigma_{y y} \sigma_{z z} \leq 1
\end{aligned}
$$




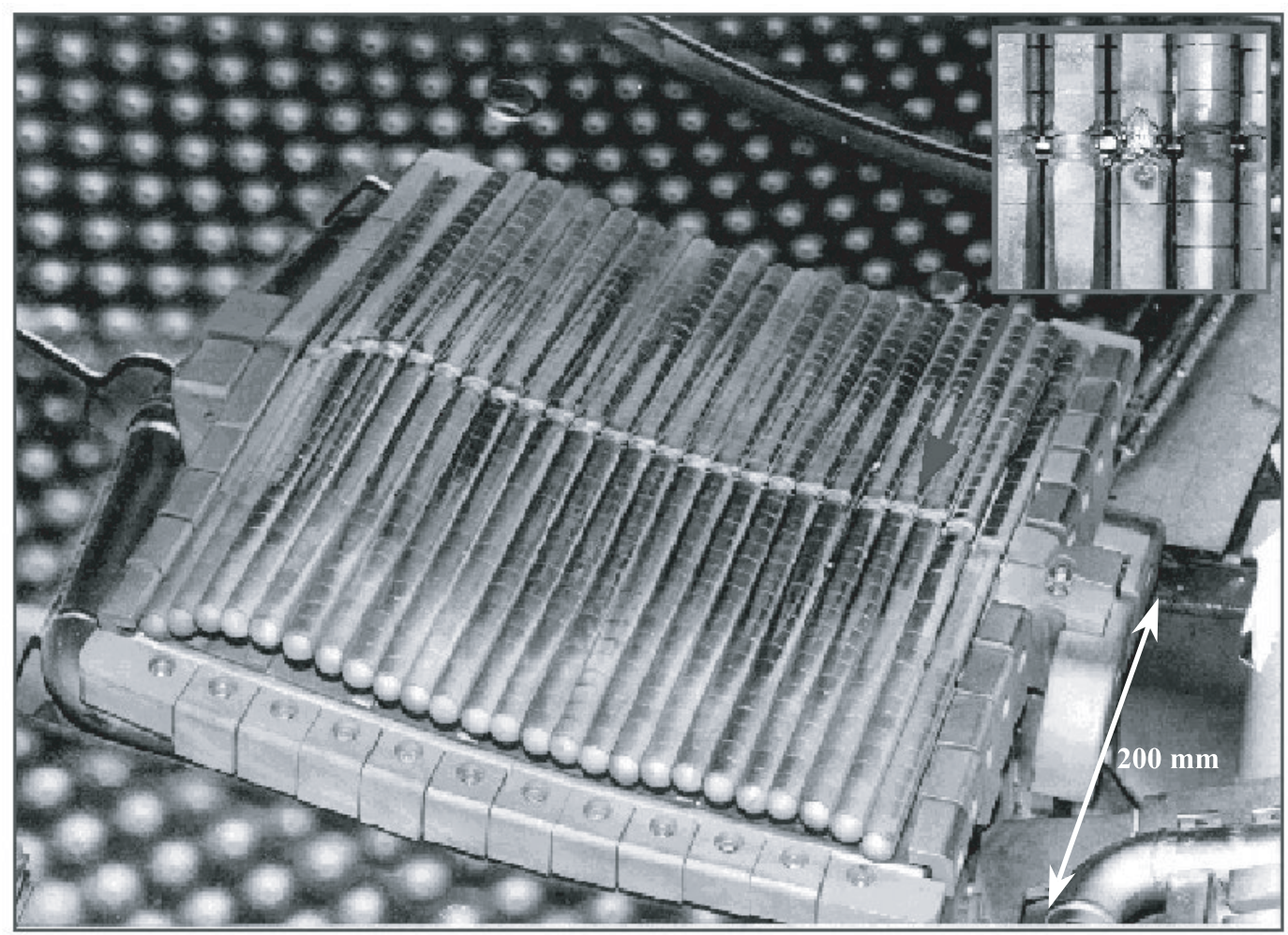

Fig. 5. Vue d'un limiteur vertical modulaire de Tore Supra $\left(1^{\mathrm{e}}\right.$ génération de CFP). La partie zoomée montre la crête du limiteur où les tubes activement refroidis et recouverts de graphite polycristallin ont été abîmés par des électrons de « run away ».

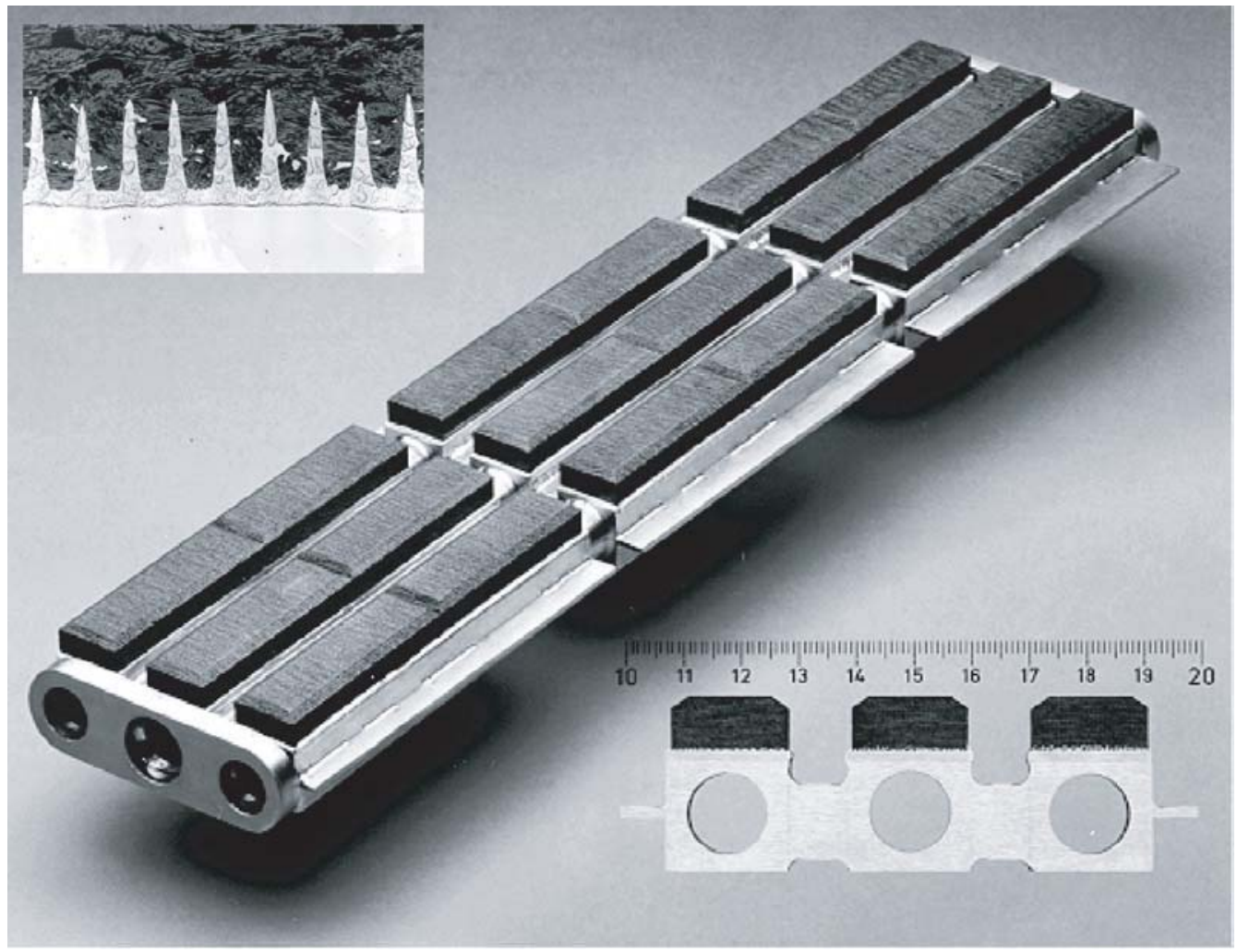

Fig. 6. Vue d'un élément modulaire de seconde génération de la première paroi de Tore Supra : on montre en haut à gauche les trous coniques de $0,5 \mathrm{~mm}$ de profondeur effectués au laser sur la surface à braser de la tuile et en bas à droite une coupe de l'élément. 


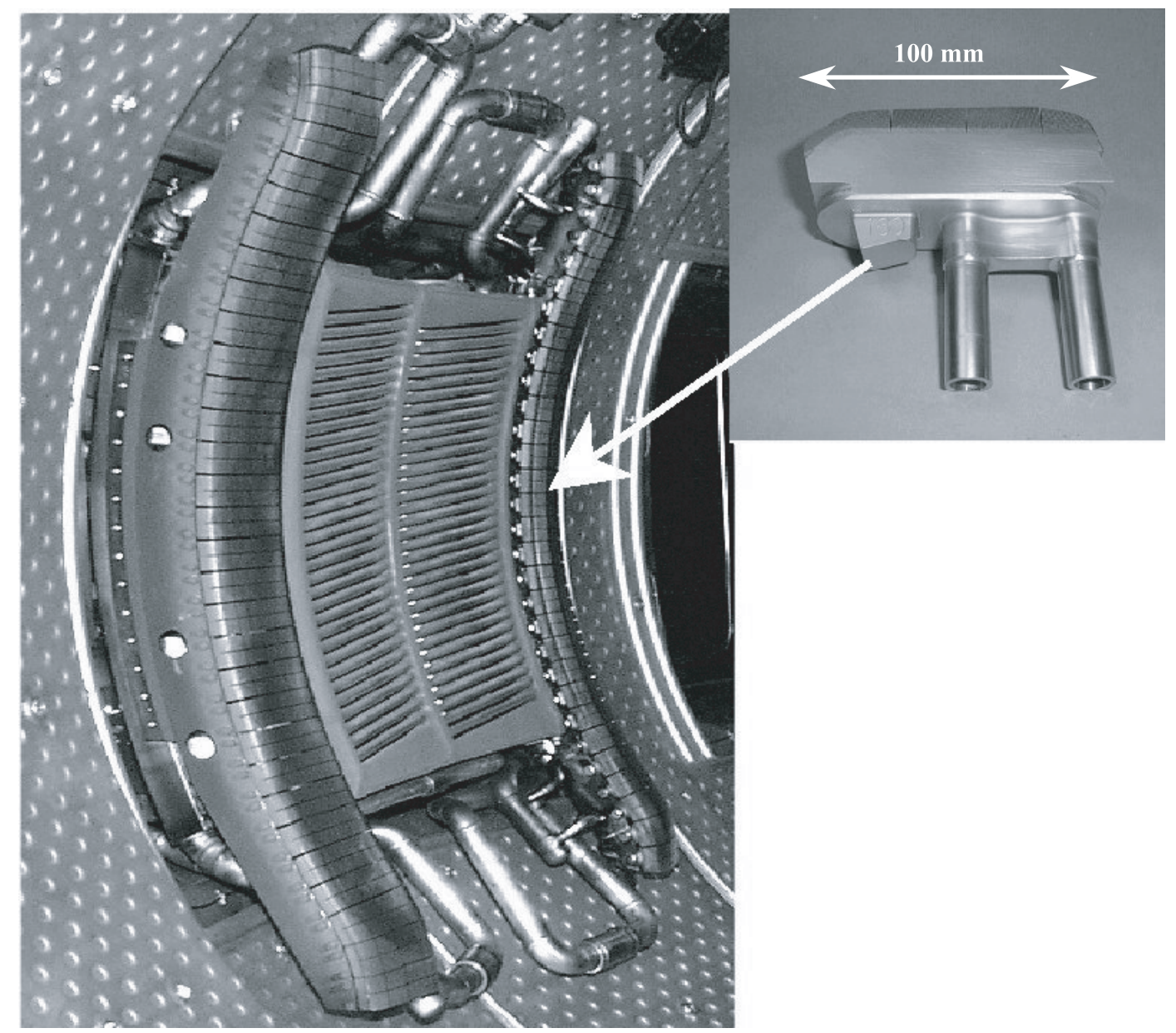

Fig. 7. Vue d'un élément de limiteur d'antenne (env. $10 \mathrm{~cm}$ de longueur) et d'une antenne avec ses 2 limiteurs formés chacun de 30 éléments activement refroidis à l'eau.

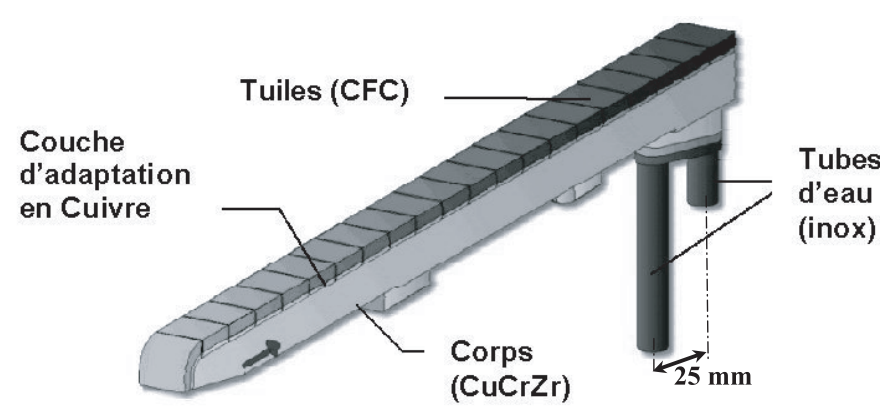

Fig. 8. Vue du composant élémentaire (aiguille) du nouveau limiteur toroidal de Tore Supra activement refroidi à l'eau (longueur $495 \mathrm{~mm})$.

avec :

$$
\begin{aligned}
& F_{11}=\frac{1}{R_{x} R_{x^{\prime}}} ; \quad F_{22}=\frac{1}{R_{y} R_{y^{\prime}}} ; \quad F_{33}=\frac{1}{R_{z} R_{z^{\prime}}} \\
& H_{11}=\left(\frac{1}{R_{x}}-\frac{1}{R_{x^{\prime}}}\right) ; \quad H_{22}=\left(\frac{1}{R_{y}}-\frac{1}{R_{y^{\prime}}}\right) ; \\
& H_{33}=\left(\frac{1}{R_{z}}-\frac{1}{R_{z^{\prime}}}\right)
\end{aligned}
$$

$$
\begin{aligned}
& C_{12}=\frac{1}{S_{x y^{2}}} ; \quad C_{13}=\frac{1}{S_{x z^{2}}} ; \quad C_{23}=\frac{1}{S_{y z^{2}}} ; \\
& F_{i j}=\frac{1}{2} \sqrt{F_{i i} F_{j j}}
\end{aligned}
$$

Les termes $R$ sont les résistances à la traction, les termes $R^{\prime}$ sont les résistances à la compression, les termes $S$ sont les résistances au cisaillement. Tous ces termes ont été identifiés par des tests en traction ou en cisaillement. Les termes $F_{i j}$ proposés ici ne sont qu'une première approche qui mériterait d'être affinée.

Ce type de critère a permis de relativement bien prédire la rupture des briquettes au cours des essais de caractérisation du joint [16]. Pour le matériau considéré (N11 fabriqué par SNECMA-SEP, Bordeaux) nous avons les valeurs données dans le tableau 1 .

De nombreux essais ont été mis en œuvre pour caractériser le joint $\mathrm{AMC} \AA[10,15]$. On trouvera des résultats synthétiques sur les figures 12 et 13. Les essais ont été menés soit de façon monotone soit en effectuant des cycles (5 chargements/déchargements croissants ou bien 30 cycles à $75 \%$ de la limite à la rupture), 21 éprouvettes ont été testées en traction et 28 éprouvettes en cisaillement. L'originalité des essais 


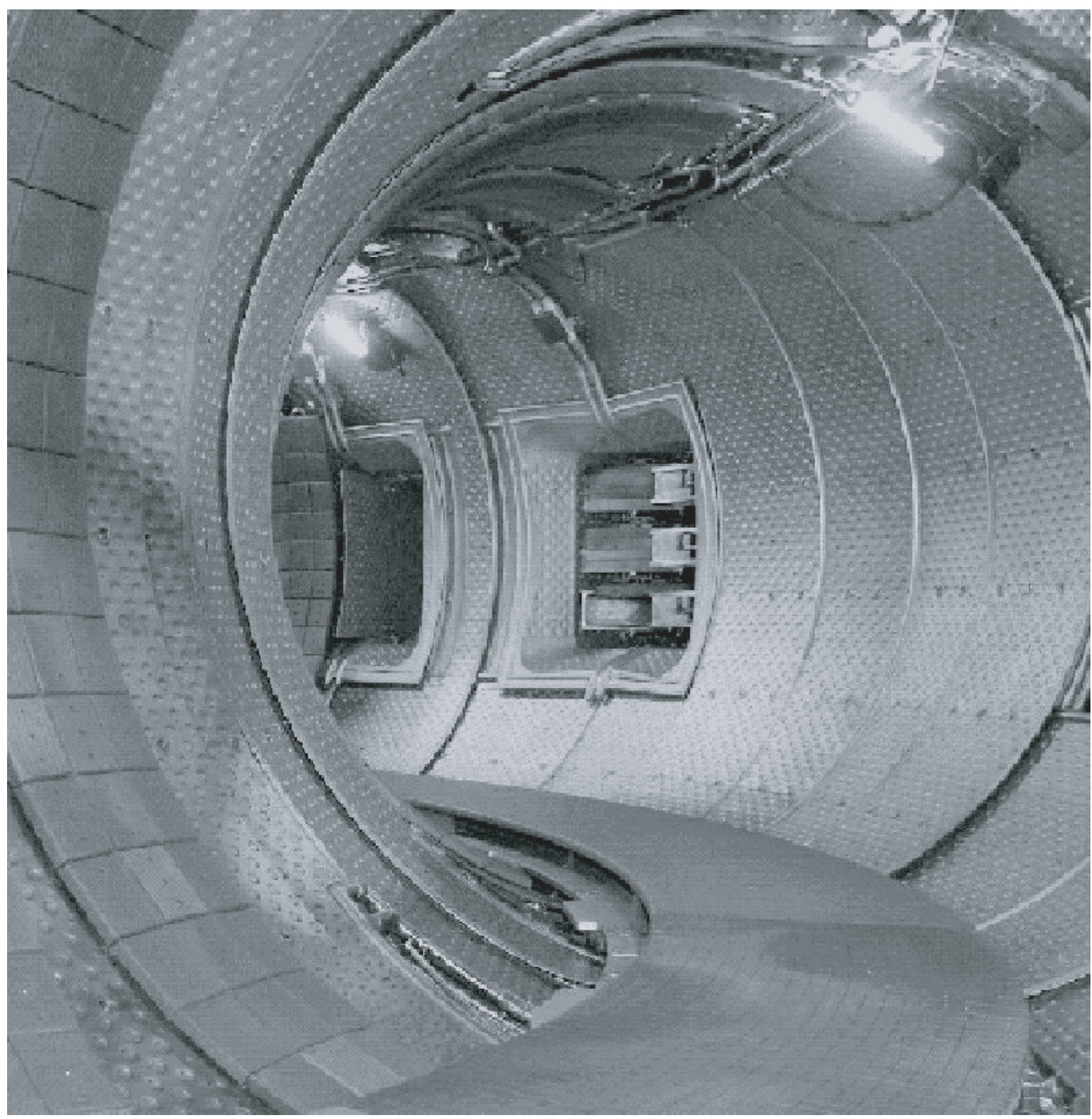

Fig. 9. Vue du nouveau limiteur toroidal formé d'environ 600 aiguilles activement refroidies à l'eau (largeur de la couronne : $495 \mathrm{~mm})$.

Tableau 1. Limites à la rupture du matériau N11 en fonction de la température.

\begin{tabular}{lccccc}
\hline & & $20{ }^{\circ} \mathrm{C}$ & $500{ }^{\circ} \mathrm{C}$ & $1000{ }^{\circ} \mathrm{C}$ & $1500{ }^{\circ} \mathrm{C}$ \\
\hline$R_{x x}=R_{y y}$ & $(\mathrm{MPa})$ & 50 & 50 & 60 & 70 \\
$R_{x x}^{\prime}=R_{y y}^{\prime}$ & $(\mathrm{MPa})$ & 100 & 100 & 120 & 140 \\
\hline$R_{z z}$ & $(\mathrm{MPa})$ & 20 & 24 & 28 & 32 \\
$R_{z z}^{\prime}$ & $(\mathrm{MPa})$ & 80 & 80 & 95 & 110 \\
\hline$S_{x y}=S_{y x}$ & $(\mathrm{MPa})$ & 20 & 20 & 24 & 28 \\
$S_{x z}=S_{y z}$ & $(\mathrm{MPa})$ & 18 & 20 & 23 & 25 \\
\hline
\end{tabular}

tient à ce qu'ils ont été effectués à froid et à chaud jusqu'à des températures de $600{ }^{\circ} \mathrm{C}$. En traction on observe que le joint résiste beaucoup moins bien que le $\mathrm{CFC}$, ce qui montre que l'accrochage du cuivre sur le CFC reste assez faible. Au contraire en cisaillement l'assemblage est beaucoup plus résistant que le CFC, en effet la structuration de la surface permet de créer un matériau intermédiaire, CFC-cuivre plus résistant que le $\mathrm{CFC}$ et dont la résistance s'accroît lors des cycles, probablement par écrouissage du cuivre. À partir de $400{ }^{\circ} \mathrm{C}$ en cisaillement c'est la couche d'adaptation en cuivre doux qui « lâche » en plastification plutôt que la liaison $\mathrm{CFC} / \mathrm{Cuivre}$, ce phénomène est particulièrement net à $600{ }^{\circ} \mathrm{C}$.

\section{Conclusion}

La liaison graphite/métal utilisée dans le Tokamak Tore Supra est une liaison intrinsèquement difficile à réaliser. Des solutions industrielles performantes ont été trouvées :

a) par brasage de briquettes en CFC sur de l'inox par l'intermédiaire d'une couche de cuivre doux pour évacuer des flux de 2 MW.m ${ }^{-2}$,

b) par un procédé original dit « $\mathrm{AMC} \AA$ » qui permet de relier une briquette en $\mathrm{CFC}$ à un support en cuivre à durcissement structural sans dépasser les $450{ }^{\circ} \mathrm{C}$ 


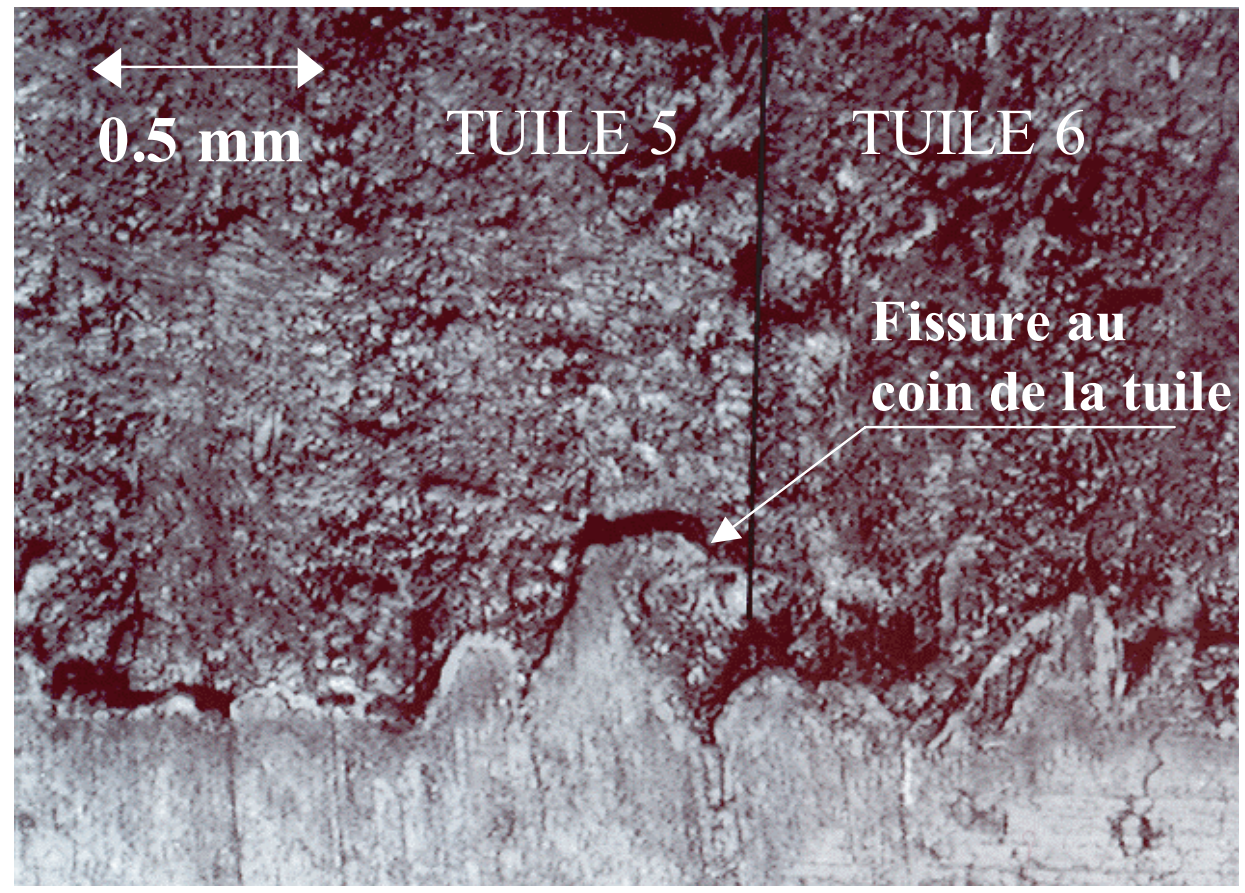

Fig. 10. Vue d'une fissure typique en mode de traction qui se développe en suivant l'interface CFC/cuivre.

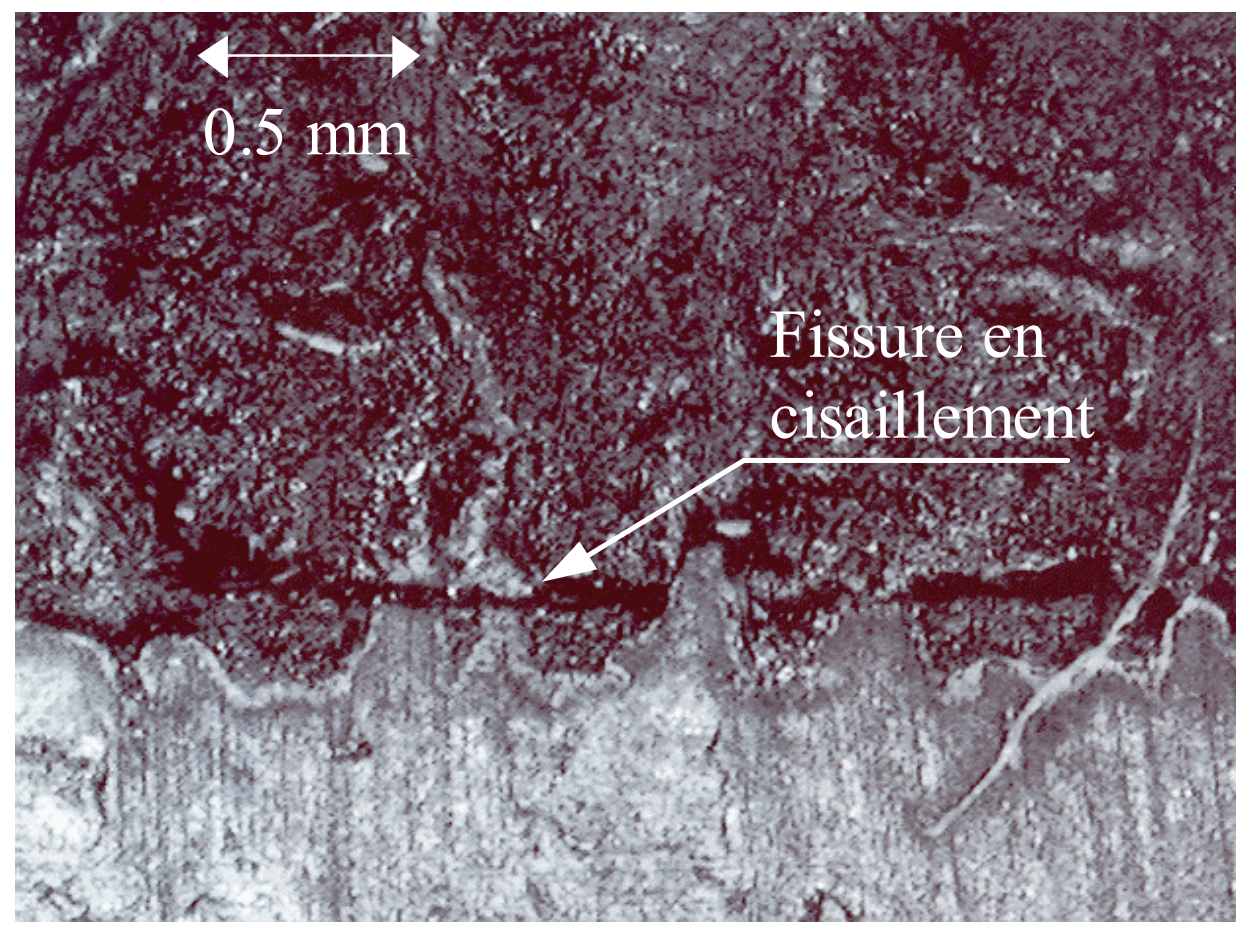

Fig. 11. Vue d'une fissure typique en mode de cisaillement qui se développe à travers les picots de cuivre.

sur le support pendant le processus de fabrication. Ce dernier assemblage permet d'évacuer des flux jusqu'à $15 \mathrm{MW} . \mathrm{m}^{-2}$.

Ces 2 types de procédés utilisent un processus de traitement par laser de la surface du CFC à assembler de façon à former un réseau de trous coniques. Au cours de la dernière fabrication de 600 éléments pour le limiteur toroïdal de Tore Supra (plus de 12000 briquettes) un taux anormal de fissuration de type traction (arrachement) s'est produit. Ce type de fissuration est bien corrélé avec les essais de caractérisation du joint $\mathrm{AMC} \cap$, en effet si ce type de joint montre une résistance au cisaillement étonnante sa résistance à la traction reste faible. Il serait souhaitable de poursuivre les études pour renforcer la jonction intime entre le cuivre et les fibres de carbone afin d'améliorer la tenue mécanique à l'arrachement. 


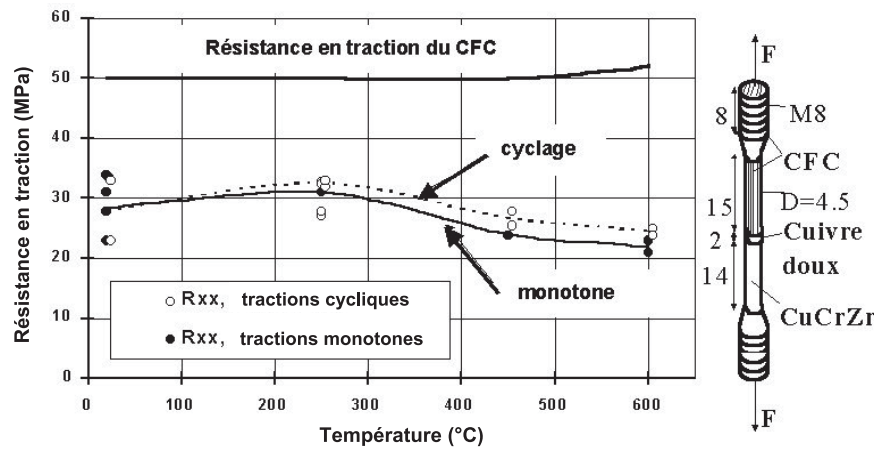

Fig. 12. Résultats des tests de traction à différentes températures sur des éprouvettes $\mathrm{CFC} /$ cuivre. La résistance en traction de l'assemblage reste très en dessous de la résistance en traction du CFC seul.

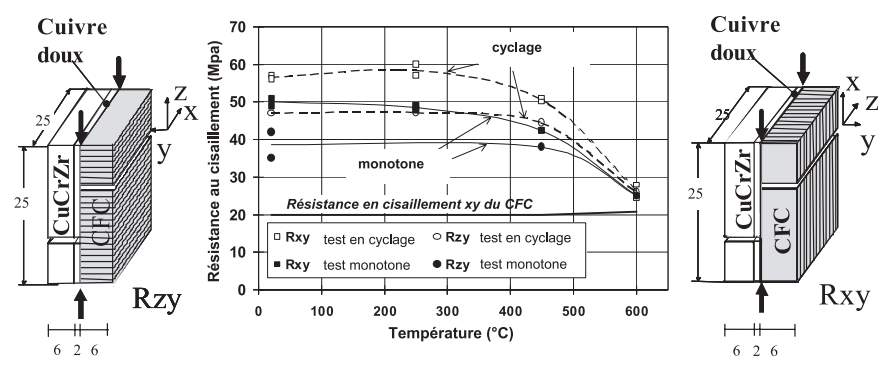

Fig. 13. Résultats des tests de cisaillement à différentes températures sur des éprouvettes $\mathrm{CFC} /$ cuivre. Le cyclage de l'éprouvette avant d'atteindre la valeur maximale améliore très sensiblement les résultats. Les valeurs obtenues sont très audessus de la résistance en cisaillement du CFC seul.

\section{Références}

[1] T. Yada, H. Koguchi, Reliability Evaluation of Joints of Ceramics and Metal (Consideration of Thermoelastic Plastic Stress Around the Interface of Jointed Dissimilar Materials), JSME Int. J. Series I, 34(2) (1991) 163-170

[2] K. Mizuno, K. Miyazawa, T. Suga, Characterization of Thermal Stresses in Ceramic/Metal Joint, J. Faculty Engng., the University of Tokyo (B) 39(4) (1988) 401412

[3] V.L. Hein, F. Erdogan, Stress Singularities in a TwoMaterial Wedge, Int. J. Fracture Mechanics 7(3) (1971) $317-330$

[4] M. Lipa, P. Chappuis, P. Deschamps, Brazed Graphite for Actively Cooled Plasma-Facing Components in Tore Supra. Description, Tests and Performances, Fusion Technology 19 (1991) 2041-2048

[5] M. Lipa, P. Chappuis, G. Chaumat, D. Guilhem, R. Mitteau, L. Plöchl, Development and fabrication of improved CFC-brazed components for the inner first wall of Tore Supra, Proc. of the 18th SOFT, Kalsruhe, Germany, 1994, pp. 455-458
[6] J. Schlosser, P. Chappuis, M. Chatelier, J.J. Cordier, P. Deschamps, L. Garampon, D. Guilhem, M. Lipa, R. Mitteau, In-service experience feedback of the Tore Supra actively cooled inner first wall, Fusion Engineering and Design 27 (1995) 203-209

[7] T. Huber, L. Plöchl, N. Reheis, J.P. Cocat, J. Schlosser, The manufacturing and testing of the toroidal pumped limiter prototype elements for Tore Supra, in Proc. 16th IEEE/NPSS/SOFE, Champaign, 1995, pp. $716-719$

[8] G. Agarici, B. Beaumont, B. Bibet, S. Bremond, J. Bucalossi, L. Colas, A. Durocher, L. Gargiulo, L. Ladurelle, G. Lombard, G. Martin, P. Mollard, First Plasma experiments in Tore Supra with a new generation of high heat flux limiter for RF antenna, Fusion Engineering and Design 49-50 (2000) 145-150

[9] J. Schlosser, P. Chappuis, M. Chatelier, A. Durocher, D. Guilhem, M. Lipa, R. Mitteau, L. Plöchl, G. Tonon, E. Tsitrone, Design, fabrication and testing of an improved High Heat Flux Element, experience feedback on steady state Plasma Facing Components in Tore Supra, Fusion Engineering and Design 39-40 (1998) 235-240

[10] J. Schlosser, P. Chappuis, A. Durocher, L. Moncel, P. Garin, Development of actively cooled components for Tore Supra Toroidal Pump limiter, Physica Scripta T91 (2001) 94-97

[11] J. Schlosser, A. Durocher, T. Huber, P. Chappuis, P. Garin, W. Knabl, B. Schedler, Material properties and consequences on the quality of Tore Supra Plasma Facing Components, J. Nuclear materials 307-311 (2002) 686-690

[12] J. Schlosser, P. Chappuis, L. Doceul, M. Chatelier, J.P. Cocat, L. Garampon, P. Garin, R. Mitteau, A. Moal, L. Plöchl, G. Tonon, E. Tsitrone, Developments for an actively cooled toroidal pump limiter for Tore Supra, in Proc. 19th Symp. of Fusion Technology (SOFT), Lisbon, Portugal, 16-20 September 1996, pp. 447-450

[13] A. Durocher, R. Mitteau, V. Paulus, J. Schlosser, Interface quality control by infrared thermography measurement, Proceedings of the 15th World Conference on Non-Destructive Testing, Roma, Italy, 15-21 Oct. 2000

[14] R. Mitteau, P. Chappuis, L. Moncel, J. Schlosser, Evidence of damage in carbon fiber composite tiles joined to a metallic heat sink under high heat flux fatigue, J. Nuclear Materials 258-263 (1998) 972-977

[15] L. Moncel, J. Schlosser, R. Mitteau, L. Plöchl, Active Metal Casting technique in Plasma Facing Components: characterization, in Proc. 20th Symp. of Fusion Technology (SOFT), Marseille, France, 7-11 September 1998, pp. $133-136$

[16] L. Moncel, Étude des mécanismes d'endommagement d'un assemblage Cuivre/Composite Carbone-Carbone sous chargement Thermomécanique, Thèse de l'université de Bordeaux I, 1999 